\title{
Hypericin as a marker for determination of tissue viability after radiofrequency ablation in a murine liver tumor model
}

\author{
MARIE VAN DE PUTTE ${ }^{1}$, HUAIJUN WANG ${ }^{2}$, FENG CHEN ${ }^{2}$, PETER A.M.DE WITTE ${ }^{1}$ and YICHENG NI $^{2}$ \\ ${ }^{1}$ Laboratory for Pharmaceutical Biology, Faculty of Pharmaceutical Sciences, ${ }^{2}$ Department of Radiology, \\ University Hospital, Faculty of Medicine, K.U. Leuven, Herestraat 49, B-3000 Leuven, Belgium
}

Received October 8, 2007; Accepted November 22, 2007

\begin{abstract}
In this proof-of-principle study, the necrosis avid agent hypericin was investigated as a potential indicator for early therapeutic response following radiofrequency ablation (RFA) of murine liver tumors. Eight mice bearing intrahepatic RIF-1 tumors were intravenously injected with hypericin $1 \mathrm{~h}$ before or $24 \mathrm{~h}$ after RFA treatment. Mice were euthanized $24 \mathrm{~h}$ after hypericin injection and excised livers were investigated by means of fluoromacroscopic and fluoromicroscopic examinations in combination with conventional histomorphology. Significant differences in hypericin fluorescence were found in necrosis, viable tumor and normal liver tissue in a decreasing order: in necrosis, mean fluorescence densities were about 5 times higher than in viable tumor and approximately 12 times higher than in normal liver $(\mathrm{p}<0.05)$. Mean fluorescence densities were not significantly different when hypericin was injected $24 \mathrm{~h}$ after or $1 \mathrm{~h}$ before RFA treatment ( $\mathrm{p}>0.05$ ). As a conclusion, hypericin features the property to specifically enhance the imaging contrast between necrotic and viable tissues and to non-specifically distinguish viable tumor from normal liver. The results suggest that hypericin offers significant potential in the early assessment of response following necrosis-inducing antineoplastic treatments such as RFA.
\end{abstract}

\section{Introduction}

The major advantage of tumor ablation therapies is their minimal invasiveness with a resultant effect comparable to that of surgical resection in the treatment of malignant tumors. Radiofrequency ablation (RFA) is one of the most commonly used, because it is associated with a higher rate of complete tumor necrosis. RFA involves the placement of an electrode-

Correspondence to: Dr Yicheng Ni, Department of Radiology, University Hospital, Faculty of Medicine, K.U. Leuven, Herestraat 49, B-3000 Leuven, Belgium

E-mail: yicheng.ni@med.kuleuven.be

Key words: necrosis, necrosis avid contrast agent, hypericin, RF ablation, liver neoplasm needle directly into the tumor that delivers destructive electrical energy to heat the tissue, resulting in $1-5 \mathrm{~cm}$ zones of in situ coagulation necrosis (1). Nonetheless, despite the increasing clinical application of RFA, tumor recurrence due to residual viable tumor foci at the radial margins of the ablated zone remains a problem (2). Therefore, assessment of RFA therapeutic responses with imaging modalities represents an important challenge. To date, there has been no accurate postprocedural imaging method available in clinic to distinguish residual tumor islets from tissue reaction to coagulation necrosis. Problems with the current imaging modalities such as CT and MR imaging are inadequate specificity for the differentiation between inflammation and incomplete ablated viable tumor, resulting in false-positive visual effects, and the inability to depict necrotic areas smaller than $3 \mathrm{~mm}(2-4)$.

In an attempt to develop more specific, direct and accurate imaging approaches, necrosis avid contrast agents (NACAs) have recently been suggested as potential contrast enhancers for therapeutic assessment of liver tumor after RFA (5). NACAs firmly label non-viable tissues and visualize them as persistent hyperintense regions on MR imaging, independent tissue origin or cause of necrosis (6).

Hypericin, a naturally occurring photosensitizer derived from St. John's Wort of the plant genus Hypericum, was recognized as a non-porphyrin necrosis avid agent, since the compound showed a peculiar affinity for irreversibly damaged ischemic or necrotic tissues (6). To date, radiolabeled derivatives of hypericin such as $\left[{ }^{123} \mathrm{I}\right]$-iodohypericin have been studied in animal models of hepatic and myocardial infarction (7). Moreover, in bulky subcutaneous tumors consisting of large areas of spontaneous necrosis, 19-fold higher activities of $\left[{ }^{123} \mathrm{I}\right]$-iodohypericin were recorded in necrosis compared to viable tumor (unpublished data). Based on such results, we believe that hypericin holds promise for the early assessment of response following necrosis inducing antineoplastic treatments such as RFA.

In this proof-of-principle study, the necrosis-avid hypericin was investigated in RFA-treated murine models with intrahepatic implantation of radiation-induced fibrosarcoma (RIF-1). Due to the excellent fluorescent properties of hypericin, we were able to obtain detailed macroscopic and microscopic fluorescence images of the RFA lesions, which were further co-localized with conventional histopathology to identify the coagulative necrotic zone, the peri-ablational untreated tumor and the normal liver. 


\section{Materials and methods}

Animals and tumor system. This animal experiment was in compliance with the current institutional regulations for use and care of laboratory animals. Subcutaneously implanted radiation-induced fibrosarcoma (RIF-1) in mice is one of the most widely used tumor models in cancer research for its biological stability, minimal immunogenicity, low metastatic potential and responsiveness to various therapeutic interventions (8). Similar to the recently reported rat model with liver implantation of rhabdomyosarcoma (R1) (9), we created RIF-1 growth in murine liver as a more clinically relevant tumor model (10) for studying the pharmacological property of hypericin and its potential use in minimally invasive tumor therapies such as radiofrequency ablation (RFA). Ten female $\mathrm{C} 3 \mathrm{H} / \mathrm{Km}$ mice (Charles River Laboratories, France) weighing 21-25 g were anesthetized with intraperitoneal injection of Nembutal ${ }^{\circledR}$ (Sanofi Sante Animale, Brussels, Belgium) at $40 \mathrm{mg} / \mathrm{kg}$ and midline laparotomized as tumor recipients. Tumor tissue $\left(1 \mathrm{~mm}^{3}\right)$, freshly harvested from a donor mouse with subcutaneous growth of RIF-1, was implanted in the left lateral liver lobe of the recipient mouse, which was allowed to recover after closure of the abdomen with layered sutures. The RIF-1 tumor growth in the liver was monitored routinely using magnetic resonance imaging (MRI) at a 1.5 Tesla clinical scanner (Sonata, Siemens, Germany) coupled with a small surface loop coil (MRI Devices Corp., Waukesha, WI, USA) under isoflurane gas anesthesia (IMS, Harvard Apparatus, Holliston, MA, USA). About 14 days after implantation, a liver mass of $0.5 \mathrm{~cm}$ in diameter, hyperintense on T2weighted or slightly hypointense on T1-weighted MR images, was regarded adequate for receiving ablation therapy (Fig. 1). One mouse died of overdose of anesthesia shortly after liver tumor implantation and one mouse was found without tumor growth, and both were eliminated from the study.

RFA procedure. Under the same anesthesia regime as that for liver tumor implantation, tumor ablation procedures were performed with laparotomy instead of percutaneous intervention in order to better steer the extent of ablation.

The liver lobe bearing RIF-1 tumor was exposed out of the incision and held gently by the operator. Being connected with a 500-kHz RF generator (RFG-3E, Radionics, Burlington, MA, USA), an 18-gauge electrode with a 1-cm uninsulated tip was inserted into the palpable RIF-1 tumor. For conducting this monopolar RFA, the electric circuit was completed through a $10 \times 8 \mathrm{~cm}$ metallic grounding pad underneath the shaved back of the mice. Under temperature control mode $\left(90^{\circ} \mathrm{C}\right)$, RF current was delivered into the tumor for $20 \mathrm{sec}$ and seized till the intratumoral temperature reached $90^{\circ} \mathrm{C}$. The RFA treated tumor and liver tissues became immediately brownish pale due to thermal coagulation (1), whereas the appearances of viable tumor and liver tissues remained unchanged. The abdominal incision in mice was closed with layered sutures after the treatments.

Hypericin preparation and administration. Hypericin was synthesized from emodin anthraquinone according to Falk et al (11). Prior to intravenous injection, hypericin was dissolved in a mixture of $25 \%$ dimethylsulfoxide (DMSO), $25 \%$

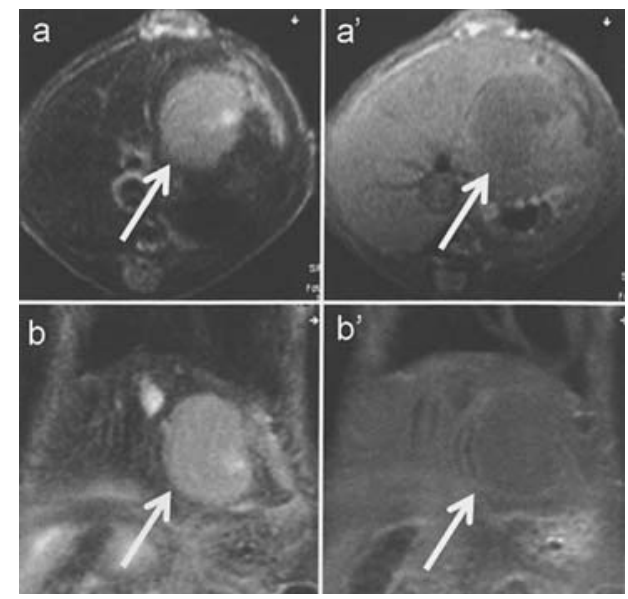

Figure 1. Monitoring liver tumor growth with magnetic resonance imaging (MRI): two weeks after implantation of RIF-1 tumor tissue chip, a spherical hyperintense and slightly hypointense liver mass of $0.5 \mathrm{~cm}$ in diameter (arrow) was detected on transverse ( $a$ and $\left.a^{\prime}\right)$ and coronal ( $b$ and $b^{\prime}$ ) T2-weighted (a and $b$ ) and T1-weighted (a' and $b^{\prime}$ ) images respectively, which was regarded adequate for receiving ablation therapy.

polyethylene glycol (PEG) 400 and water at a concentration of $2 \mathrm{mg} / \mathrm{ml}$. The solution was injected in the tail vein at a dose of $10 \mathrm{mg} / \mathrm{kg}$, either $1 \mathrm{~h}$ before $(\mathrm{n}=4)$, or $24 \mathrm{~h}$ after $(\mathrm{n}=4)$ RFA of the liver tumor.

Macroscopic and microscopic fluorescence examinations. Mice were euthanized by an intraperitoneally injected overdose of phenobarbital $24 \mathrm{~h}$ after hypericin injection. Livers were excised and photographed under a $\mathrm{UV}_{365}$ and Tungsten light to assess gross distribution of hypericin in treated tumor and surrounding liver. Subsequently, treated tumors surrounded by normal liver were dissected and immediately mounted in medium (Tissue Tek embedding medium, Miles Inc., Elkhart, IN, USA) and immersed in liquid nitrogen. Different cryostat sections (5 $\mu \mathrm{m}$ slices) were taken from each tumor and examined by fluorescence microscopy (Axioscope 2 plus) equipped with a light-sensitive charge-coupled device digital camera (AxioCam HR, Carl Zeiss, Göttingen, Germany) to acquire fluorescence images. To visualize hypericin, the Zeiss filter set 14 (excitation: BP 510-560 nm, emission: LP $590 \mathrm{~nm}$ ) was used. Eventually, tissue slices were stained with hematoxylin and eosin (H\&E) for conventional light microscopy and photomicrography.

Imaging quantitative analyses. Quantitative results of fluoromicroscopic images were obtained by manually drawing regions of interest (ROI), 8 ROIs per tumor $(n=8)$, in ablated and viable tissue areas. A distinction between ablated tissue, viable tumor and normal liver was made based on the histological examination of the H\&E stained slices. Mean fluorescence densities, corrected for background, were obtained by means of a KS imaging software system (Carl Zeiss, Vision, Hallbergmoos, Germany). Ratios of fluorescence densities in the different regions were then calculated for each condition. Statistical analysis was performed using Prism 4.00 (GraphPad Software, San Diego, CA, USA). A two-way ANOVA with Bonferroni test was performed and $\mathrm{p}$-values of $\mathrm{p}<0.05$ were considered statistically significant. 

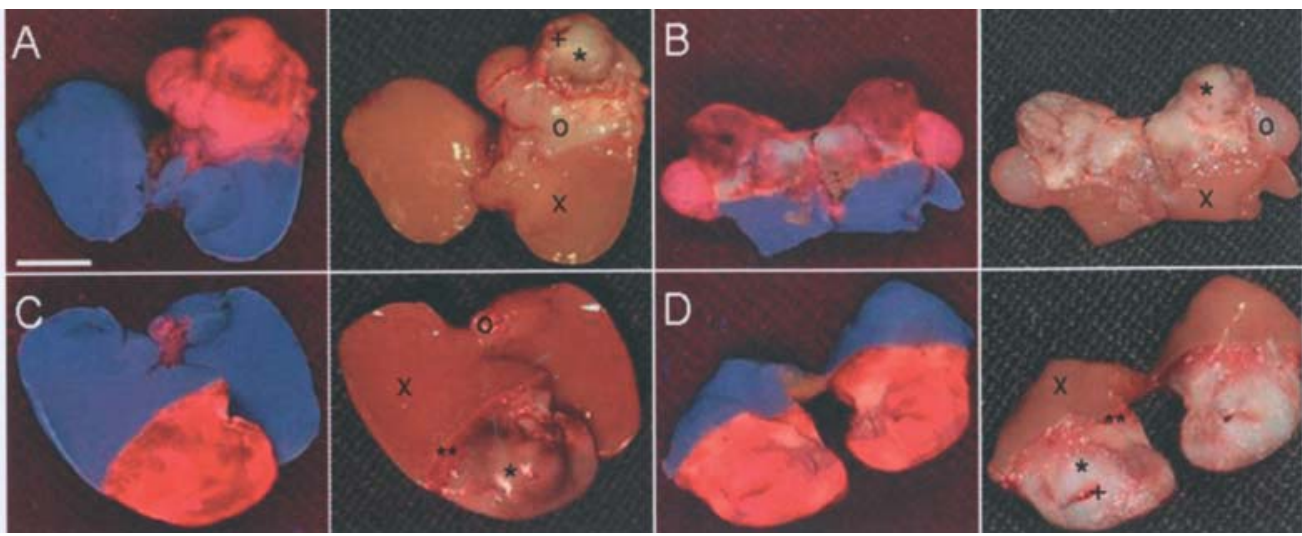

Figure 2. Macroscopic photographs taken under UV light and corresponding photographs under Tungsten light of livers bearing RIF-1 tumors treated with radiofrequency ablation (RFA). Mice were i.v. injected with $10 \mathrm{mg} / \mathrm{kg}$ hypericin at $24 \mathrm{~h}$ after (A and B) or $1 \mathrm{~h}$ before (C and D) RFA treatment. All mice were sacrificed at $24 \mathrm{~h}$ after hypericin injection and digitally photographed. Ablated tumor $(*)$, viable tumor (O), normal liver (x), ablated liver $(* *)$ and electrode track $(+)$. Scale bar, $0.5 \mathrm{~cm}$

\section{Results}

Macroscopic fluorescence imaging. On macroscopic photographs taken under UV light (Fig. 2), a perfect match was found when compared with the Tungsten light macroscopic photographs. The pale coagulative tumor necrosis, the fishflesh-like residual viable tumor, and normal liver parenchyma showed different levels of fluorescence intensity, which enabled an apparent demarcation of the different zones. Especially at the border between necrotic and viable tissue, an outstanding cut-off fluorescence signal was seen.

The effect was prominent either when mice received hypericin after RFA treatment (Fig. 2A and B), or when hypericin was given before (Fig. 2C and D). During the $24 \mathrm{~h}$ following hypericin injection, the normal liver was predominantly cleared from hypericin in contrast to viable tumor tissue, which displayed moderately elevated hypericin fluorescence intensity at $24 \mathrm{~h}$ p.i. Liver transections (Fig. 2B and D) show a highly fluorescent ablated zone which was intermingled with non-fluorescent areas, corresponding with thermally fixed no-entry zones. In Fig. 2C and D, the tumor, at the core of the RFA-induced necrotic lesion, was completely encompassed by a thick rim composed of necrotic liver tissue, suggesting a radical tumor ablation.

Microscopic fluorescence imaging. Fluorescence photomicrography confirmed the macroscopic view of three zones with different fluorescence intensities, i.e. necrotic tissue, viable tumor and normal liver (Fig. 3A and B). No-entry zones for hypericin (Fig. 3C) were found in coagulated areas surrounding the electrode track. The bulk tumor received the highest temperatures, causing preservation of the tissue via heat-induced instantaneous denaturation of enzymatic proteins (1). Only the borderline between the ablated tissue and normal liver, showed high uptake of hypericin, resulting in a fluorescent rim surrounding the no-entry zone. Fig. 3D shows the border between this no-entry zone and the surrounding fluorescent rim. On H\&E stains, this no-entry zone appeared almost intact, showing no signs of necrotic cell death, i.e. the ghost phenomenon (1), whereas the rim had a typical appearance of eosinophilic coagulation tissue.
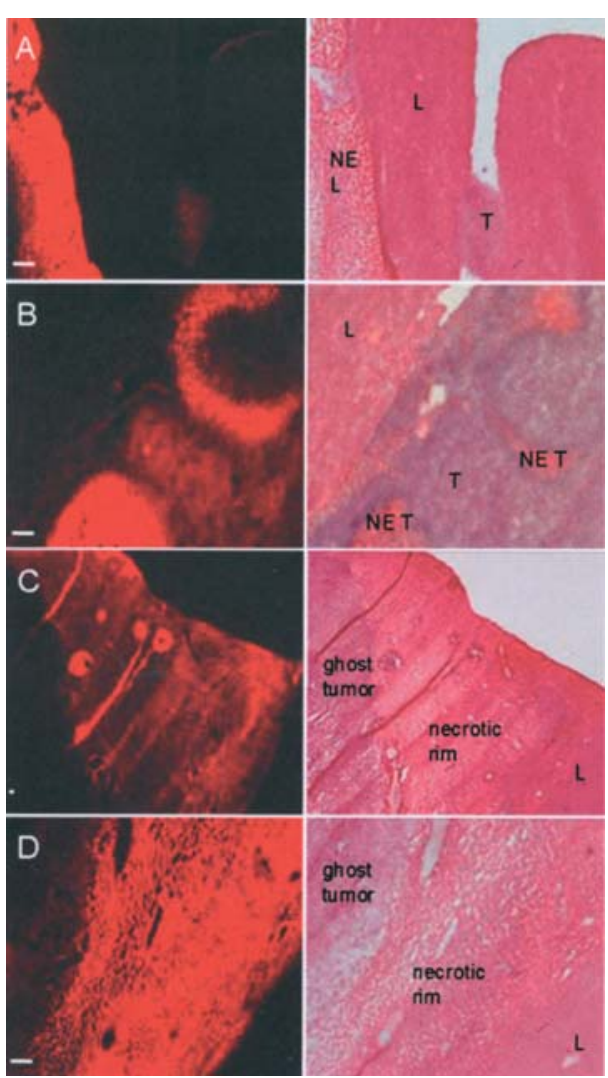

Figure 3. Fluorescence photomicrographs and corresponding hematoxylin and eosin stains of $5 \mu \mathrm{m}$ slices of livers bearing RIF-1 tumors treated with radiofrequency ablation (RFA). Mice were i.v. injected with $10 \mathrm{mg} / \mathrm{kg}$ hypericin at $24 \mathrm{~h}$ after (A and B) or $1 \mathrm{~h}$ before (C and D) RFA treatment. All mice were sacrificed at $24 \mathrm{~h}$ after hypericin injection. Ablated tumor (NE T), viable tumor (T), normal liver (L) and ablated liver (NE L). Scale bar, $100 \mu \mathrm{m}$.

Quantitative outcomes of fluorescence microscopy. Ratios of mean fluorescence densities in different areas of ablated tissue, viable tumor and normal liver are shown in Table I. Overall, ratios indicate 3 different levels of fluorescence density in necrotic tissue, viable tumor and normal liver in a decreasing order: in necrosis, mean fluorescence densities were about 5 times higher than in viable tumor and $\sim 12$ times higher than in normal liver $(\mathrm{p}<0.05)$. Mean fluorescence densities were not 
Table I. Ratios of mean fluorescence densities in different regions of fluoromicroscopic images taken from $5 \mu \mathrm{m}$ tumor sections.

\begin{tabular}{|c|c|c|c|c|c|}
\hline & NE tissue & NE tissue & NE tissue & Viable T & Ghost T \\
\hline$\Delta \mathrm{T}(\mathrm{h})$ & Viable T & Ghost T & Normal L & Normal L & Normal L \\
\hline \multirow[t]{2}{*}{24} & $3.76 \pm 1.55$ & $16.42 \pm 0.65$ & $12.93 \pm 4.72$ & $4.70 \pm 1.09$ & $0.97 \pm 0.40$ \\
\hline & ns & a & ns & ns & ns \\
\hline 1 & $6.08 \pm 0.36$ & $6.26 \pm 3.08$ & $11.88 \pm 1.81$ & $3.43 \pm 2.01$ & $2.70 \pm 0.39$ \\
\hline
\end{tabular}

significantly different when hypericin was injected $24 \mathrm{~h}$ after or $1 \mathrm{~h}$ before RFA treatment ( $\mathrm{p}>0.05$ ), except for ghost tissue, which displayed 3.95-fold higher fluorescence densities when hypericin was injected $1 \mathrm{~h}$ before, suggesting a prior entry of hypericin before the tissue was thermally fixed.

\section{Discussion}

An ideal tumor RFA lesion contains a center of coagulated tumor, surrounded by a zone of coagulated normal tissue as safety margin, without residual tumor islets in the margins of the ablated area (12). In practice, RFA lesions however are not always perfectly spherical (13). The heat-sink effect of nearby blood vessels in the perfused organ may be responsible for dissipation of the heat and frequently causes a lesion asymmetrically smaller than expected, resulting in incomplete ablation and local tumor recurrence (14). Early detection of a residual or locally recurrent tumor by means of post-procedural imaging is therefore critical for the outcome of RFA. On the other hand, the main problem with current short-term followup CT or MR imaging techniques within 3 months after treatment is the inability to distinguish residual viable tumor in the periphery of the treated lesion from reactive hyperemia (4,15-17). This inflammatory reaction, due to thermal injury, results in a peripheral rim enhancement that envelopes the ablated lesion during 3 months after treatment. Since the viable tumor islets in the peripheral rim cannot be readily distinguished, as long as the enhanced rim is present, it is impossible to diagnose the peripheral residual or regrowing tumor. As a consequence, with current contrast-enhanced CT and MR imaging, estimation of response to RFA can be made only relatively late following treatment through evidence of absent tumor regrowth (18).

The main objective of this preclinical study was to evaluate the possibilities of hypericin for use as an early therapeutic response indicator following necrosis inducing treatments such as RFA. For this purpose, we designed a murine RIF-1 liver tumor model by implanting a small tissue chip of a subcutaneously grown tumor in the liver. Unlike other animal studies that recruited subcutaneously implanted tumors, this murine hepatic tumor model may better mimic clinical conditions including visceral blood perfusion, parenchymal tumor growth and relevant microenvironment, hence providing more clinically pertinent information for cancer diagnosis and therapy. Other advantages are successful tumor growth in $84 \%$ of cases, rapid tumor growth in about 2 weeks, high survival rate above $90 \%$ till the end of experiment, minimal metastatic potential and uncomplicated implantation procedure.

As demonstrated in the present study, significant differences in hypericin fluorescence were found in the three areas of interest, i.e. necrosis, viable tumor and normal liver tissue. Thus, the 3 different tissue components could be visually stratified, even as early as $24 \mathrm{~h}$ after RFA. In an earlier study (unpublished data) concerning the mechanism of hypericin's avidity for necrosis, it was found that hypericin specifically stained necrotic tissue by compound-dependent interactions with necrotic cellular debris, whereas viable tissues (e.g. tumor and liver) were non-specifically labeled to a lesser extent. Thus, the observed minimal but persistent enhancement in viable tumor $24 \mathrm{~h}$ p.i. can be explained mainly by the nonspecific uptake of hypericin by the tumor cells and/or stroma and a lack of the mechanisms for biliary excretion and/or lymphatic drainage in the tumor, similar to the phenomenon reported with MRI hepatobiliary contrast agents (19). By contrast, hypericin was substantially cleared from the normal liver within $24 \mathrm{~h}$ due to functioning hepatic metabolic activities and biliary excretion.

On the other hand, the thermal effects of RFA involve an important vascular shutdown in the ablated zone (1), which limits perfusion and subsequent diffusion of hypericin throughout the entire necrotic lesion. As a result, hypericin will enter the necrotic zone through residual functioning blood vessels in the vicinity, followed by its diffusion into the necrotic debris driven by the concentration gradient, causing an evolving rim of intense fluorescence. However, this process cannot completely explain why only the periphery of RFA lesions is stained at $24 \mathrm{~h}$ p.i. In contrast to traditional coagulation necrosis, where enzymatic degradation plays a predominant role, RFA-induced necrosis is characterized by an area of thermal fixation due to instantaneous denaturation of cytosolic enzymes upon elevated temperature (1). This thermally fixed tissue is therefore not promptly degraded into cellular debris and, although it involves dead tissue, it looks almost identical to viable tissue with conventional histological staining 
techniques (H\&E). This phenomenon has been described in the literature as the 'ghost' phenomenon or 'thermal fixation' $(1,5)$. It has already been suggested earlier that in vivo tissue degradation is required before interaction with hypericin can take place (6). Yet, degradation of thermally fixed tissue requires a time-consuming process of days to weeks, during which the center of RFA-induced coagulative necrosis will be infiltrated by immune cells. Upon digestion of the dead tissue by enzymatic reactions, the necrotic debris will be absorbed and replaced by granulation tissue, though extensive lesions may result in incomplete absorption and fibrous encapsulation due to limited penetration of immune cells (1).

In this study, two approaches were conducted by injecting hypericin before or after RFA treatment. When hypericin was injected $1 \mathrm{~h}$ before, maximal blood concentrations were achieved about the time of the RFA treatment (20), so that upon ablation an outstanding extravasation took place in the necrotic rim surrounding the thermally fixed ghost tissue. The latter showed 3.95-fold higher fluorescence densities when compared to ghost tissue in the $24 \mathrm{~h}$ condition, due to partial extravasation of hypericin prior to thermal fixation. After $24 \mathrm{~h}$, normal liver was substantially cleared from hypericin and viable residual tumor was non-specifically enhanced, resulting in three distinguishable zones and a striking fluorescence cut-off at the border between the ablated zone and viable normal tissue on both macroscopic and microscopic level. The approach with injection of hypericin $1 \mathrm{~h}$ prior to ablation has the advantage that shorter time intervals are required between RFA treatment and imaging.

Although this study has demonstrated the proof-of-principle of hypericin as a marker for early therapeutic assessment after RFA, a number of issues have to be settled before this compound can be applied in patients. First, fluorescence imaging requires invasive procedures in order to display RFA lesions in visceral organs. Applying a laparoscopic approach, which has been increasingly adopted in clinical practice, may reduce its invasiveness. Secondly, real non-invasive imaging can only be realized by tagging hypericin with a marker for a particular imaging modality, for instance, a gadolinium chelate for MR imaging and a radioactive tracer for nuclear imaging, though the latter may suffer from insufficient spatial resolution. It remains to be investigated whether hypericin derivatives, designed as a contrast agent for MRI, produce signals strong enough for the stratification of tumors in non-necrotic, necrotic and severe necrotic regions. Lastly, despite the extensive clinical use of hypericin as a photosensitizer for photodynamic diagnosis and therapy of certain superficial cancers (21) and as a constituent of Hypericum extracts that are used clinically as a mild antidepressant, hypericin is characteristic of potential phototoxicity. This may particularly limit the use for MR visualization and fluorescence imaging, where higher amounts are injected. Local drug delivery in combination with a transcatheter interventional procedure may significantly increase the local concentration while reducing its dosage, hence minimizing any dose-related side effects. For nuclear imaging, the phototoxicity of hypericin is unimportant since only very small amounts of the compound are administered.

In conclusion, the results of the present study suggest that hypericin offers significant potential in the early assessment of response following necrosis-inducing antineoplastic treatments such as RFA. Hypericin features the property to specifically enhance the imaging contrast between necrotic and viable tissues and to non-specifically distinguish viable tumor from normal liver. We believe that our results have shown sufficient promise to justify continued research. Labeling hypericin to extend its use to MR imaging could be a first step in this direction.

\section{Acknowledgements}

This study was supported by grants awarded by Fonds voor Wetenschappelijk Onderzoek-Vlaanderen (FWO Vlaanderen), a Geconcerteerde Onderzoeksactie (GOA) of the Flemish Government, and an EU project Asia-Link CfP 2006-Europe Aid/123738/C/ACT/Multi-Proposal No. 128-498/111.

\section{References}

1. Ni Y, Mulier S, Miao Y, Michel L and Marchal G: A review of the general aspects of radiofrequency ablation. Abdom Imaging 30: 381-400, 2005.

2. Guan Y, Sun L, Zhou X, Li X and Zheng X: Hepatocellular carcinoma treated with interventional procedures: CT and MRI follow-up. World J Gastroenterol 10: 3543-3548, 2004.

3. King A, Tse G, Ahuja A, Yuen E, Vlantis A, To E and van Hasselt A: Necrosis in metastatic neck nodes: diagnostic accuracy of CT, MR imaging, and US. Radiology 230: 720-726, 2004.

4. Goldberg S: Science to practice: can we differentiate residual untreated tumor from tissue responses to heat following thermal tumor ablation? Radiology 234: 317-318, 2005.

5. Ni Y, Chen F, Mulier S, Sun X, Yu J, Landuyt W, Marchal G and Verbruggen A: Magnetic resonance imaging after radiofrequency ablation in a rodent model of liver tumor: tissue characterization using a novel necrosis avid contrast agent. Eur Radiol 16: 1031-1040, 2006.

6. Ni Y, Bormans G, Chen F, Verbruggen A and Marchal G: Necrosis avid contrast agents: functional similarity versus structural diversity. Invest Radiol 40: 526-535, 2005.

7. Ni Y, Huyghe D, Verbeke K, De Witte P, Nuyts J, Mortelmans L, Chen F, Marchal G, Verbruggen A and Bormans G: First preclinical evaluation of mono-[ $\left.{ }^{123} \mathrm{I}\right]$ iodohypericin as a necrosisavid tracer agent. Eur J Nucl Med Mol Imaging 33: 595-601, 2006.

8. Twentyman P, Brown J, Gray J, Franko A, Scoles M and Kallman R: A new mouse tumor model system (RIF-1) for comparison of end-point studies. J Natl Cancer Inst 64: 595-604, 1980.

9. Chen F, Sun X, De Keyzer F, Yu J, Peeters R, Coudyzer W, Vandecaveye V, Bosmans H, van Hecke P, Landuyt W, Hermans R, Marchal G and Ni Y: A rodent liver tumor model with implanted rhabdomyosarcoma: characterization using magnetic resonance, microangiography and histopathology. Radiology 239: 554-562, 2006.

10. Wang H, van de Putte M, Chen F, De Keyzer F, Jin L, Yu J, Marchal G, De Witte P and Ni Y: Murine liver implantation of radiation-induced fibrosarcoma: characterization with MR imaging, microangiography and histopathology. Eur Radiol (In press).

11. Falk H, Meyer $\mathrm{J}$ and Oberreiter $\mathrm{M}$ : A convenient semisynthetic route to hypericin. Monatsh Chem 124: 339-341, 1993.

12. Dodd G III, Frank M, Aribandi M, Chopra S and Chintapalli K: Radiofrequency thermal ablation: computer analysis of the size of the thermal injury created by overlapping ablations. Am J Roentgenol 177: 777-782, 2001.

13. Mulier S, Ni Y, Miao Y, Rosiere A, Khoury A, Marchal G and Michel L: Size and geometry of hepatic radiofrequency lesions. Eur J Surg Oncol 29: 867-878, 2003.

14. Goldberg S, Grassi C, Cardella J, Charboneau J, Dodd G III, Dupuy D, Gervais D, Gillams A, Kane R, Lee F Jr, Livraghi T, McGahan J, Phillips D, Rhim H and Silverman S: Image-guided tumor ablation: standardization of terminology and reporting criteria. Radiology 235: 728-739, 2005. 
15. Antoch G, Vogt F, Veit P, Freudenberg L, Blechschmid N, Dirsch O, Bockisch A, Forsting M, Debatin J and Kuehl H: Assessment of liver tissue after radiofrequency ablation: findings with different imaging procedures. J Nucl Med 46: 520-525, 2005.

16. Dromain C, De Baere T, Elias D, Kuoch V, Ducreux M, Boige V, Petrow P, Roche A and Sigal R: Hepatic tumors treated with percutaneous radio-frequency ablation: $\mathrm{CT}$ and MR imaging follow-up. Radiology 223: 255-262, 2002.

17. Kim T, Moon W, Cha J, Goo J, Lee K, Kim K, Lee J, Han J, Weinmann $\mathrm{H}$ and Chang $\mathrm{K}$ : VX2 carcinoma in rabbits after radiofrequency ablation: comparison of MR contrast agents for help in differentiating benign periablational enhancement from residual tumor. Radiology 234: 423-430, 2005.

18. Kim C, Choi D, Lim H, Kim S, Lee W, Kim M, Lee J, Jeon Y, Lee J, Lee S and Lim J: Therapeutic response assessment of percutaneous radiofrequency ablation for hepatocellular carcinoma: utility of contrast-enhanced agent detection imaging. Eur J Radiol 56: 66-73, 2005.
19. Ni Y and Marchal G: Enhanced magnetic resonance imaging for tissue characterization of liver abnormalities with hepatobiliary contrast agents: an overview of preclinical animal experiments. Top Magn Reson Imaging 9: 183-195, 1998.

20. Chen B, Xu Y, Roskams T, Delaey E, Agostinis P, Vandenheede J and De Witte P: Efficacy of antitumoral photodynamic therapy with hypericin: relationship between biodistribution and photodynamic effects in the RIF-1 mouse tumor model. Int J Cancer 93: 275-282, 2001.

21. Dolmans D, Fukumura D and Jain R: Photodynamic therapy for cancer. Nat Rev Cancer 3: 380-387, 2003. 